American Journal of Pharmacology and Toxicology 4(1): 12-16, 2009

ISSN 1557-4962

(C) 2008 Science Publications

\title{
The Effects of Recombinant Human Erythropoietin and Tamoxifen on Growth and Angiogenesis of Mammary Tumor in Sprague-Dawley Rat
}

\author{
${ }^{1}$ A.K. Sairah, ${ }^{1,2}$ A. Rasedee, ${ }^{2}$ O. Sheikh, ${ }^{3}$ R. Rozita and ${ }^{1,2}$ Nagi ALHaj \\ ${ }^{1}$ Department of Bioscience, University Putra Malaysia, 43400 Serdang, Selangor Darul Ehsan \\ ${ }^{2}$ Faculty of Veterinary Medicine, University Putra Malaysia, 43400 \\ Serdang, Selangor Darul Ehsan, Malaysia \\ ${ }^{3}$ Faculty of Medicine and Health Science, University Putra Malaysia, \\ 43400 Serdang, Selangor Darul Ehsan, Malaysia
}

\begin{abstract}
Problem statement: Erythropoietin receptor (EPOR) has been detected in breast cancer cells and speculated to be involved in cancer growth, viability and angiogenesis. This has risen suspicious that EPO administration may enhance the severity of cancer. Approach: This study was undertaken to determine the effects of rHuEPO, Tamoxifen and their combination on the growth and angiogenesis of mammary tumor. Female Sprague-Dawley rats were induced to develop mammary tumor through xenograft technique by inoculating $6 \times 10^{5}$ LCM 2388 cells. Results: Recombinant human erythropoietin, Tamoxifen and Tamoxifen-rHuEPO combination were administered weekly for four weeks and size of tumors was measured weekly. Blood was also collected weekly and serum separated and subjected to ELISA for Matrix Metalloproteinases 2 (MMP-2) and Vascular Endothelial Growth Factor (VEGF), quantification. The animals were sacrificed at the end of experiment and tumor masses excised for histopathological analysis. Results showed no significance difference in the growth of mammary tumor of rats that received rHuEPO compared to the control rats. Interestingly, the combination of rHuEPO and Tamoxifen produced approximately $90 \%$ tumor regression from the initial size compared to Tamoxifen alone which showed 70\% tumor regression. Quantification of serum angiogenic factors, MMP2 and VEGF of rHuEPO treatment group showed lower concentrations than the control group. Conclusion/Recommendations: Among all groups, Tamoxifen-treated group showed the lowest concentration of the angiogenic factors. The mitotic index of the tumor from all groups were observed to be at low frequency (G1). In conclusion, rHuEPO did not produce any significant promoting effect either on tumor growth, angiogenesis or tumor cell proliferation.
\end{abstract}

Key words: Recombinant human erythropoietin, tamoxifen, matrix metalloproteinases vascular endothelial growth factor, mammary gland tumor, University Putra Malaysia

\section{INTRODUCTION}

Cancer progression is influenced by multiple factors including induction of tumor angiogenesis. Understanding tumor angiogenesis and growth at its early stages can provide new insights into the mechanisms relevant to tumor progression and metastasis and facilitate the development of novel antiangiogenic therapies. According to Folkman ${ }^{[1]}$ many regulatory molecules released by tumor and/or host cells mediate the induction of tumor angiogenesis and that constitute potential targets for anti-angiogenic therapy. Vascular Endothelial Growth Factor (VEGF), an important regulator of both physiologic and pathologic angiogenesis, has been successfully targeted in preclinical tumor models as well as in clinical trials involving cancer patients. However, the benefits of antiangiogenic therapy can be limited by the redundant mechanisms of angiogenesis control, a problem that may be overcome by targeting multiple angiogenic pathways or the use of broad spectrum angiogenic inhibitors.

Recombinant human erythropoietin (rHuEPO) has been used to treat anemia in cancer patient since early $1990 \mathrm{~s}^{[4]}$. Generally, the use of rHuEPO in clinical practice has greatly enhanced the management of anemia and substantially improved patient quality of life. However, there is some doubt concerning the safety of rHuEPO administration in anemic cancer patient, since EPO Receptor (EPOR) has been detected

Corresponding Author: Rasedee Abdullah, Faculty of Veterinary Medicine, Department of Pathology and Microbiology, University Putra Malaysia, Serdang 43400, SDE, Malaysia Tel: 603-89441582 
in human breast cancer cells and that EPO is involved in growth, viability and angiogenesis of the malignant tumors ${ }^{[9]}$. This has raised the suspicious that usage of rHuEPO in malignancy may stimulate growth of tumor and thus further enhances severity of the cancer. However, evidence to support this growth-promoting effect has been inconclusive as it was also shown that rHuEPO has no effect on tumor growth or angiogenesis ${ }^{[3]}$. Most of the previous reports on the effect of rHuEPO on tumor growth were performed in vitro. The present study was undertaken to determine the in vivo effects of rHuEPO on mammary gland tumor using a rat model. The gross changes, tumor size, quantification of circulating angiogenic factors and cell proliferation analyses were performed to consequently ascertain the effects of rHuEPO administration on tumor growth.

\section{MATERIALS AND METHODS}

Animal management and induction of mammary tumor development: Twenty-four female SpragueDawley rats aged six weeks were used in this study. The animals were acclimatized for 3-4 days before tumor induction. A total of $6 \times 10^{7}$ LCM 2388 rat mammary gland tumor cells were inoculated subcutaneously into mammary fat pad of the rats. The rats were monitored daily to observe the occurrence of tumor and treatments were initiated only after the tumors have reached sizes of $1.0-1.5 \mathrm{~cm}$ at any diameter.

Experimental design: Commercial rHuEPO (Eprex $\left.{ }^{\circledR}\right)$ at a dose of $60 \mathrm{IU}$ and Tamoxifen of $20 \mathrm{mg} \mathrm{mL}^{-1}$ in soy oil were used in this study. Four groups of rats consisting of six rats per group were assigned as follows: Group I (60 IU rHuEPO), Group II (20 mg Tamoxifen), Group III (60 IU rHuEPO+20 mg Tamoxifen) and Group IV (1mL normal saline solution). After tumor detection (week 1) Tamoxifen were given orally while rHuEPO and normal saline for control group were administered via intraperitoneal injections. All rats were inspected daily, but weighed weekly. Tumor size measurement also was performed weekly. The tumors were measured in $\mathrm{mm} \times \mathrm{mm}$ (length $\mathrm{x}$ width) using a digital caliper.

Sample collection: One milliliter blood was collected weekly via cardiac puncture and allowed to clot at room temperature before centrifuging at $10,000 \times \mathrm{g}$ for $10 \mathrm{~min}$ for serum separation.

VEGF and MMP-2 analysis: The concentrations of serum VEGF and MMP-2 were determined by a commercial ELISA Kits: Quantikine ${ }^{\mathrm{TM}}$ Rat VEGF Immunoassay (R\&D Systems, USA) and Quantikine ${ }^{\mathrm{TM}}$ Rat MMP-2 Immunoassay (R and D Systems, USA) respectively. All samples were analyzed in duplicates. ELISA plates were read using a microplate reader (Tecan, Austria). The standard curve of VEGF and MMP-2 were generated to determine their concentrations in the samples.

Histopathological analysis: The rats were sacrificed at the end of experiment and tumor masses excised and fixed in $10 \%$ buffered formalin, dehydrated in series of alcohol, embedded in paraffin wax and sectioned at $5 \mu \mathrm{m}$. The sections then were stained with Hematoxylin and Eosin ( $\mathrm{H}$ and $\mathrm{E})$. Mitotic index analysis of the tumor was performed by counting the number of mitoses in 10 random high-power fields at $400 \mathrm{x}$ magnification (10HPF; $x 400$ ) and the highest count in the 10 fields was taken as the Mitotic Activity Index (MAI). The indices were classified into grade 1 (GI) with 0-4 mitotic figures and Grade 2 (G2) with 5 or more mitotic figures according to the method described by Ouchi et al..$^{[5]}$.

\section{RESULTS}

Xenograft technique for mammary tumor induction: The xenograft technique was shown to rapidly induce tumor growth within a short duration of 14 days after inoculation of the tumor cells.

Effect of rHuEPO on mammary tumor growth: The changes in mammary tumor size for all treatment groups are shown in Fig. 1. Significant increase $(p<0.05)$ in mammary tumor size after 4 weeks was observed in the control group which showed 100\% increase from the initial size. Grossly, there is no significance difference in mammary tumor growth between the group receiving $\mathrm{rHuEPO}$ and the control group. However, the group that received Tamoxifen and the Tamoxifen-rHuEPO combination demonstrated significant tumor regression. The Tamoxifen treatment group at week 5 showed tumor regression by more than $70 \%$ from the initial size. Treatment with a combination of Tamoxifen and rHuEPO showed better effects with approximately $90 \%$ regression from the initial tumor size.

Serum angiogenic factors (MMP-2 and VEGF): The serum MMP-2 concentrations in the four treatment groups during the experiment are presented in Fig. 2. Generally, the control group showed the highest MMP2 activity during the study that reached peak value at 


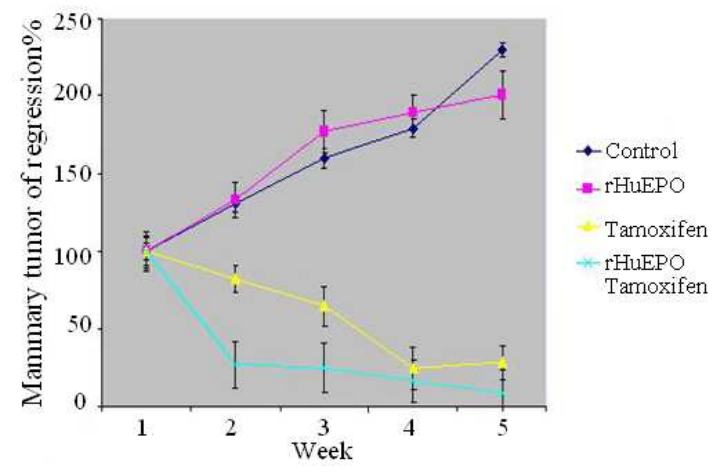

Fig. 1: Weekly tumor regression percentage of mammary tumor of the four treatment groups. (Data are mean and bars are SEM)

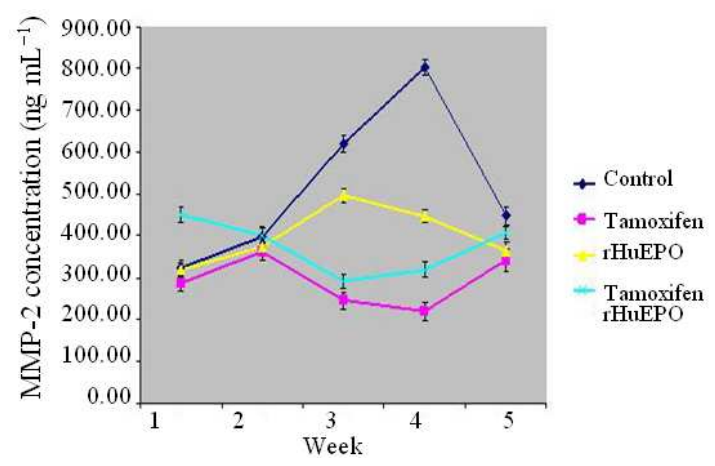

Fig. 2: Weekly changes of serum MMP-2 concentration of the four treatment group. (Data are mean and bars are SEM)
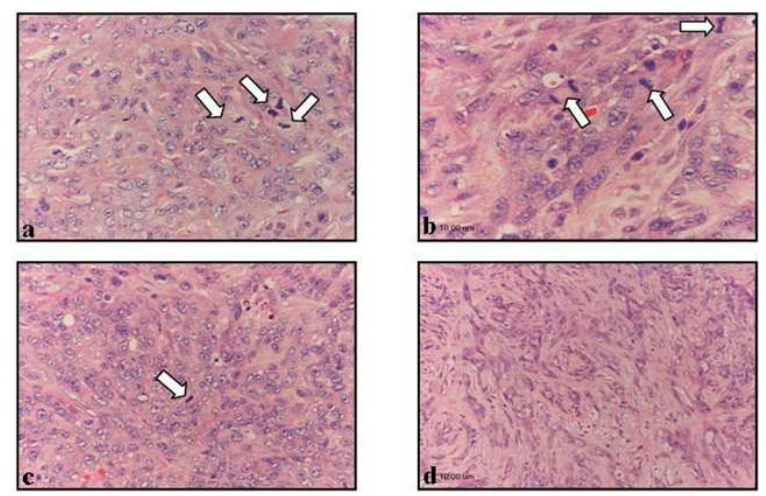

Fig. 3: Mammary tumor section from rats showing mitotic figures (arrows) (H\&E, 400x) (a) Control, (b) rHuEPO treatment, (c) Tamoxifen treatment, d) Tamoxifen-rHuEPO treatment

week 4, while Tamoxifen treatment group showed the lowest activity throughout the period of experiment.
The MMP-2 activity in the rHuEPO treatment group was observed to have a similar pattern as the control group; however, the level is much lower. Meanwhile, the group receiving Tamoxifen-rHuEPO combination showed almost the same MMP-2 concentrations at weeks 1 and 5.

The serum VEGF concentration was extremely low in these rats. In the control rats, the VEGF concentration at week 3 was $27.14 \mathrm{pg} \mathrm{mL}^{-1}$ and not detectable at other times. For rats that received rHuEPO, the VEGF activity was detected only at week 5 at $4.72 \mathrm{pg} \mathrm{mL}^{-1}$. While for the Tamoxifen group, the VEGF concentration was $3.14 \mathrm{pg} \mathrm{mL}^{-1}$ at week 3 . No VEGF activity was detected in the samples of the Tamoxifen-rHuEPO combination group.

Mitotic index analysis: Figure 3 represents histological sections of mammary tumor tissues from the treatment groups showing mitotic figures. Very few mitotic figures were observed in the control and rHuEPO treatment groups. The mitotic figures were rarely seen in the tumors of Tamoxifen and Tamoxifen-rHuEPO treatment groups. In general the mitotic index in all groups was of the same grade, G1.

\section{DISCUSSION}

The xenograft mammary tumor model seems to be reliable and promising and had resulted in rapid occurrence of the tumor. This xenograft model would afford an opportunity for cancer researchers to conduct in vivo study within a very short period. This method of tumor induction is recommended because it minimizes the risk associated with the conventional induction method using carcinogens. Furthermore, this xenograft mammary tumor seems to be similar to human breast cancers with respect to histopathological characteristics (unpublished data). This xenograft model then was used to evaluate the effects of rHuEPO and Tamoxifen administration on the growth of the mammary tumor. Generally, it was found that rHuEPO treatment did cause any promoting effect on the tumor growth as the tumor progression pattern between rHuEPO treatment and control groups was quite similar throughout the study period. It is speculated that rHuEPO does not activate the proliferation signaling pathways for tumor growth. In fact, our study suggests that rHuEPO may have beneficial effects in delaying tumor growth. This is evident in the study when rats treated with rHuEPO finally had smaller mammary tumors than the control group.

Administration of Tamoxifen resulted significant reduction in tumor growth reflected by size regression 
of more than $70 \%$. It is obvious that Tamoxifen administration does inhibit tumor progression, although there are toxicity effects associated with the drug. Although Tamoxifen has been effectively used in the treatment of the breast cancer for more than three decades, the specific molecular mechanisms underlying its effect on tumor regression remain unclear. However, Tamoxifen can inhibit cell proliferation and induce apoptosis by activating the caspase pathways ${ }^{[7]}$. Caspases are cysteine proteases present in the cytosol in inactive forms and generally the procaspases must be first proteolytically cleaved at specific aspartate residues to become the active caspases ${ }^{[2]}$. Thus, it is believed that in Tamoxifen-treated rats, the caspase pathway is activated and concurrently the highly mitogenic capability of the tumor cells may be suppressed by the deactivation of cell proliferation signaling pathways, specifically the MAPKs pathway.

Interestingly, the combination of rHuEPO and Tamoxifen administration has resulted in a greater reduction in tumor size compared to treatment with Tamoxifen alone. The regression of the tumor size was observed to be approximately $90 \%$ from the initial size. This indicates that rHuEPO and Tamoxifen cooperate in enhancing inhibitory and antiproliferative effects on the tumor growth. It is also speculated that the signals for activation of caspase pathways are much greater than those for activation of MAPK pathway which lead to apoptosis rather than proliferation. However, the exact mechanism by which the combination of rHuEPO and Tamoxifen exert its anti-roliferative effects in mammary tumor is yet to be elucidated.

Previously Ribatti ${ }^{[6]}$ has suggested that EPO may promote tumor growth by stimulating angiogenesis through MMP-2. However, the present study has found that $\mathrm{rHuEPO}$ treatment may not stimulate angiogenesis in the mammary tumor since the MMP-2 concentration in rHuEPO-treated rats was lower than in the control rats. This points to the possible role of rHuEPO in the inhibition of angiogenesis in the tumor tissue. Among the treatment groups, the control group showed the highest serum MMP-2 concentration. The MMP-2 showed an increasing pattern as the result of rHuEPO administration, peaking at week 4 before declining towards the end of experiment. This increasing pattern may reflect an active pathological angiogenesis which promoted tumor growth. However, by week 4 when the tumor had attained maximum size, the MMP-2 concentrations began to wane, may be because the tumor had exhausted its capability to sustain angiogenesis. For the Tamoxifen and TamoxifenrHuEPO treatment groups the serum MMP-2 concentrations were lower compared to control.
Tamoxifen alone seemed to have the greatest effect in the reduction of serum MMP-2 concentration. The results suggest that Tamoxifen suppresses angiogenic activity which consequently inhibits tumor growth, while rHuEPO did not potentiate this effect.

Another circulating parameter for angiogenesis, VEGF was also determined in this study. Unfortunately, the VEGF concentrations in these samples were too low to be consistently detectable. However, in samples where VEGF was detected, the highest was in the control followed in order by rHuEPO treatment and Tamoxifen treatment groups. No VEGF was detected in the serum of the Tamoxifen-rHuEPO treatment group. High serum VEGF concentration in control group reflects the pathologic angiogenesis associated with active tumorgenesis. It is postulated that during tumorgenesis, the VEGF transcription is up regulated and sustained to support the angiogenesis.

Histopathologically the proliferation in the tumor tissue is indicated by increase in mitotic index. In all groups including the rHuEPO treatment rats, the mitotic index was observed at a low grade, G1. Contrary to earlier suggestions ${ }^{[9]}$ this study suggests that rHuEPO administration within our study period did not cause significant tumor cell proliferation. The low mitotic index in Tamoxifen and Tamoxifen-rHuEPO treatment groups could presumably be attributed to their inhibitory effects on the tumor, also evident by the $90 \%$ tumor size regression as the result of their administration.

\section{CONCLUSION}

In conclusion, rHuEPO does not produce any significant promoting effect on tumor growth, proliferation or angiogenesis. These results also suggest that rHuEPO is safe to be used in cancer tumor patients, if administered concurrently with Tamoxifen.

\section{REFERENCES}

1. Folkman, J., 2006. Antiangiogenesis in cancer therapy-endostatin and its mechanisms of action. Exp. Cell Res., 312: 594-607. PMID: 16376330

2. Hakem, R. and L. Harrington, 2005. Cell Death. In: Ian the Basic Science of Oncology, Tannock, F., R.P. Hill, R.G. Bristow and L. Harrington (Eds.). McGraw Hill Companies, ISBN-13: 9780824722487, pp: 194-204.

3. Hardee, M.E., J.P. Kirkpatrick, S. Shan, S.A. Synder, Z. Vujaskovic, Z.N. Rabbani, M.W. Dewirst and K.L. Blackwell, 2005. Human recombinant erythropoietin (rEpo) has no effect on tumor growth or angiogenesis. Br. J. Cancer, 93: 1350-1355. PMID: PMC1891087 
4. Nowrousian, M.R., 1998. Recombinant human erythropoietin (rhEPO) in the prevention and treatment of chemotherapy-induced anaemia. Med. Oncol., 15: 141-144. ISSN 1357-0560

5. Ouchi, K., T. Sugawara, H. Ono, T. Fujiya, Y. Kamiyama, Y. Kakugawa, J. Mikuni, H. Yamanani, S. Komatsu and A. Horikoshi, 2000. Mitotic index is the best predictive factor for survival of patients with resected hepatocellular carcinoma. Digest. Surger., 17: 42-48. DOI: 10.1159/000018799

6. Ribatti, D., M. Presta, A. Vacca, R. Ria, R. Giuliani, P. Dell'Era, L. Roncali and F. Dammacco, 1999. Human erythropoietin induces a pro-angiogenic phenotype in cultured endothelial cells and stimulates neovascularisation in vivo. Blood, 93: 2627-2636. PMID: 10194442
7. Mandlekar, S., V. Hebbar, K. Christov and A.N. Tony Kong, 2000. Pharmacodynamics of Tamoxifen and its 4-hydroxy and N-desmethyl metabolites: Activation of caspases and induction of apoptosis in rat mammary tumors and in human breast cancer cell lines. Cancer Res., 60: 6601-6606. PMID: 11118041.

8. Sturk, C. and D. Dumont, 2005. Angiogenesis. In: The Basic Science of Oncology, Tannock, I.F., R.P. Hill, R.G. Bristow and L. Harrington (Eds.). McGraw Hill Companies, ISBN-13: 9780824722487, pp: 231-248.

9. Yoshiko, Y., F. Yoshihiko, M. Takuya, K. Satoshi, H. Satoshi, T. Akira, O. Mie, H. Mitsuhiro, M. Terunuga, O. Kazuhiro, F. Hiroyoshi, N. Yukio, S. Hitoshi and U. Hiroshi, 2003. Erythropoietin regulates tumor growth of human malignancies. Carcinogenesis, 24: 1021-1029. PMID: 12807756 